\title{
$D$-SIMPLE RINGS AND PRINCIPAL MAXIMAL IDEALS OF THE WEYL ALGEBRA
}

\author{
YVES LEQUAIN* \\ Instituto de Matemtica Pura e Aplicada, IMPA, Estrada Dona Castorina 110, \\ 22460-320 Rio de Janeiro RJ, Brazil \\ e-mail: ylequain@impa.br
}

DANIEL LEVCOVITZ ${ }^{\circledast}$

Departamento de Matemática, Instituto de Ciências Matemáticas e de Computação, Universidade de São Paulo - Campus de São Carlos, Caixa Postal 668, 13560-970 São Carlos SP, Brazil

e-mail:lev@icmc.usp.br

and JOSÉ CARLOS DE SOUZA JUNIOR ${ }^{\dagger}$

Departamento de Matemática, Instituto de Ciências Matemáticas e de Computação, Universidade de São Paulo - Campus de São Carlos, Caixa Postal 668, 13560-970 São Carlos SP, Brazil

e-mail: jcsouzajr@rantac.com.br

(Received 6 May, 2004; accepted 11 March, 2005)

\begin{abstract}
We prove that if the order-one differential operator $S=\partial_{1}+$ $\sum_{i=2}^{n} \beta_{i} \partial_{i}+\gamma$, with $\beta_{i}, \gamma \in K\left[x_{1}, \ldots, x_{n}\right]$, generates a maximal left ideal of the Weyl algebra $A_{n}(K)$, then $S$ does not admit any Darboux differential operator in $K\left[x_{1}, \ldots, x_{n}\right]\left\langle\partial_{2}, \ldots, \partial_{n}\right\rangle$; hence in particular, the derivation $\partial_{1}+\sum_{i=2}^{n} \beta_{i} \partial_{i}$ does not admit any Darboux polynomial in $K\left[x_{1}, \ldots, x_{n}\right]$. We show that the converse is true when $\beta_{i} \in K\left[x_{1}, x_{i}\right]$, for every $i=2, \ldots, n$. Then, we generalize to $K\left[x_{1}, \ldots, x_{n}\right]$ the classical result of Shamsuddin that characterizes the simple linear derivations of $K\left[x_{1}, x_{2}\right]$. Finally, we establish a criterion for the left ideal generated by $S$ in $A_{n}(K)$ to be maximal in terms of the existence of polynomial solutions of a finite system of differential polynomial equations.
\end{abstract}

2000 Mathematics Subject Classification. Primary 13N15, Secondary 16S32, $13 \mathrm{~N} 10$.

1. Introduction. Let $A_{n}(K)=K\left[x_{1}, \ldots, x_{n}\right]\left\langle\partial_{1}, \ldots, \partial_{n}\right\rangle$ be the $n$-th Weyl algebra over a field $K$ of characteristic zero (here $\partial_{n}$ denotes the usual derivation $\frac{\partial}{\partial x_{n}}$ ). Lately, there has been a lot of research done on the principal maximal left, or right, ideals of $A_{n}(K)$. (Recall that if $\tau$ is the standard involution of $A_{n}(K)$ and $S A_{n}(K)$ is a principal maximal right ideal, then $A_{n}(K) \tau(S)$ is a principal maximal left ideal of $A_{n}(K)$. Therefore, finding principal maximal right ideals of $A_{n}(K)$ is the same as finding principal maximal left ideals of $\left.A_{n}(K)\right)$.

The first author to address this problem was Stafford who exhibited a family of principal maximal right ideals of $A_{n}(\mathbb{C})$. In this way he gave the first counterexamples

\footnotetext{
* Partially supported by "PRONEX" - Commutative Algebra and Algebraic Geometry - Brazil.

$\circledast$ Partially supported by CNPq, Brazil, grant 300407/89-0.

$\dagger$ Supported by FAPESP grant 99/00240-2, Brazil.
} 
to the conjecture that every simple module over $A_{n}(\mathbb{C})$ should be holonomic. (Since $\frac{A_{n}(\mathbb{C})}{S A_{n}(\mathbb{C})}$ is simple but not holonomic if $n \geq 2$ ). (See [11]). Later on, Berstein and Lunts proved that, in a certain sense, the generic operator of $A_{n}(\mathbb{C})$ generates a maximal left ideal (see [1] and [8]). Nevertheless, the examples discovered by Stafford were of a different kind of the generic ones of Berstein and Lunts.

Stafford's examples were generalized by Coutinho in [3]. Starting with a certain type of simple derivation $d$ of $K\left[x_{1}, \ldots, x_{n}\right]$, he was able to give a suitable perturbation of $d$, say $d+\gamma, \gamma \in K\left[x_{1}, \ldots, x_{n}\right]$, such that the left ideal $A_{n}(K)(d+\gamma)$ is maximal. Here, for the first time, we see that there might be a connection between $d$-simplicity of $K\left[x_{1}, \ldots, x_{n}\right]$ and maximality of the left ideal of $A_{n}(K)$ generated by $d+\gamma$.

One of the objectives of this paper is to address the following question:

Question. Let $d=\partial_{1}+\alpha_{2} \partial_{2}+\cdots+\alpha_{n} \partial_{n}$ be a derivation of $K\left[x_{1}, \ldots, x_{n}\right]$ with $\alpha_{i} \in K\left[x_{1}, \ldots, x_{n}\right]$ for every $i=2, \ldots, n$. Suppose that there exists an element $\gamma \in$ $K\left[x_{1}, \ldots, x_{n}\right]$ such that the left ideal $A_{n}(K)(d+\gamma)$ is maximal. Then, is $d$ a simple derivation of $K\left[x_{1}, \ldots, x_{n}\right]$ ?

In Section 3, we obtain a positive answer to this question for the class of derivations that satisfy the following extra condition:

$$
\alpha_{i} \in K\left[x_{1}, \ldots, x_{i}\right] \text {, for every } i=2, \ldots, n \text {. }
$$

This is obtained as a consequence of two results that are interesting in their own right. The first one is that, even when the extra condition $(*)$ is not satisfied, the derivation $d$ does not admit any Darboux polynomial in $K\left[x_{1}, \ldots, x_{n}\right]$. The second one is a general result on derivations: if $d=\partial_{1}+\alpha_{2} \partial_{2}+\ldots+\alpha_{n} \partial_{n}$ is a derivation of $K\left[x_{1}, \ldots, x_{n}\right]$ that satisfies condition $(*)$, then $d$ is a simple derivation if (and only if) $d$ does not admit any Darboux polynomial in $K\left[x_{1}, \ldots, x_{n}\right]$. For the latter result, an example due to Goodearl and Warfield shows that the condition $(*)$ is not superfluous.

Another objective also treated in section 3 is to generalize to $K\left[x_{1}, \ldots, x_{n}\right]$, for a certain family of derivations (which we call Shamsuddin derivations), the result of Shamsuddin that characterizes the simple linear derivations of $K\left[x_{1}, x_{2}\right]$ in terms of the existence of a polynomial solution for a certain finite system of differential polynomial equations. We use our criterion to exibit new examples of simple derivations of $K\left[x_{1}, \ldots, x_{n}\right]$.

In Section 4, for a Shamsuddin derivation $d=\partial_{1}+\sum_{i=2}^{n}\left(a_{i} x_{i}+b_{i}\right) \partial_{i}$, with $a_{i}, b_{i} \in$ $K\left[x_{1}\right]$ for $i=2, \ldots, n$ and satisfying the condition $a_{i} \neq a_{j}$ for every $i \neq j$, we establish a criterion for the left ideal generated by $d+\gamma$ in $A_{n}(K)$ to be maximal in terms of the existence of polynomial solutions of a finite system of differential polynomial equations. This generalizes and strengthens a result of Bratti and Takagi for $A_{2}(d+\gamma)$ (see [2]). We give an example to show that the condition $a_{i} \neq a_{j}$ for every $i \neq j$ is not superfluous.

In section 2, we prove a general theorem, part of which is needed to obtain the results of section 3 . We prove that if the order-one differential operator $S=$ $\partial_{1}+\sum_{i=2}^{n} \beta_{i} \partial_{i}+\gamma$, with $\beta_{i}, \gamma \in K\left[x_{1}, \ldots, x_{n}\right]$, generates a maximal left ideal of the Weyl algebra $A_{n}(K)$, then $S$ does not admit any Darboux differential operator in $K\left[x_{1}, \ldots, x_{n}\right]\left\langle\partial_{2}, \ldots, \partial_{n}\right\rangle$. We show that the converse is true when $\beta_{i} \in K\left[x_{1}, x_{i}\right]$ for every $i=2, \ldots, n$.

Throughout this paper, $K$ will be a field of characteristic zero and $x_{1}, \ldots, x_{n}$ some indeterminates over $K$. 
If $d$ is a derivation of a $\operatorname{ring} B$, an ideal $I$ of $B$ is said to be a $d$-ideal if $d(I) \subseteq I$. The ring $B$ is said to be $d$-simple if its only $d$-ideals are (0) and (1); we shall also say that $d$ is a simple derivation of $\mathrm{B}$. A derivation $d$ of $K\left[x_{1}, \ldots, x_{n}\right]$ is said to be a Shamsuddin derivation if $d=\partial_{1}+\alpha_{2} \partial_{2}+\ldots+\alpha_{n} \partial_{n}$ where $\alpha_{i}=a_{i} x_{i}+b_{i}$, with $a_{i}, b_{i} \in K\left[x_{1}\right]$ for every $i=2, \ldots, n$.

If $S$ is an operator in $A_{n}(K)$, an element $R \in K\left[x_{1}, \ldots, x_{n}\right]\left\langle\partial_{2}, \ldots, \partial_{n}\right\rangle \backslash K$ is called a Darboux operator of $S$ in $K\left[x_{1}, \ldots, x_{n}\right]\left\langle\partial_{2}, \ldots, \partial_{n}\right\rangle$ if

$$
[S, R] \in K\left[x_{1}, \ldots, x_{n}\right] R .
$$

In particular, if $S=d$ is a derivation of $K\left[x_{1}, \ldots, x_{n}\right]$ and $R=f$ is a polynomial in $K\left[x_{1}, \ldots, x_{n}\right] \backslash K$, we say that $f$ is a Darboux polynomial of $d$ if

$$
[d, f]=d(f) \in K\left[x_{1}, \ldots, x_{n}\right] f .
$$

Equivalently, $f$ is a Darboux polynomial of $d$ if $(f)$ is a proper non-zero $d$-ideal of $K\left[x_{1}, \ldots, x_{n}\right]$.

The authors would like to thank Cydara Ripoll whose careful reading of a preliminary version of the paper helped them to improve the proof of Theorem 2.8. We also thank S.C.Coutinho and the referee for many suggestions and comments on this paper.

2. Principal maximal left ideals and darboux differential operators. Let $K$ be a field of characteristic zero and let $A_{n}=A_{n}(K)=K\left[x_{1}, \ldots, x_{n}\right]\left\langle\partial_{1}, \ldots, \partial_{n}\right\rangle$ be the Weyl algebra in $\mathrm{n}$ variables over the field $K$. Recall that $A_{n}(K)$ has generators $\partial_{i}, x_{j}$, for $1 \leq i, j \leq n$, satisfying the relations $\left[\partial_{i}, x_{j}\right]:=\partial_{i} x_{j}-x_{j} \partial_{i}=\delta_{i j}$ and other commutators being zero.

Let $A_{n-1}$ be the $K$-subalgebra of $A_{n}(K)$ generated by $x_{i}$ and $\partial_{i}$, for $2 \leq i \leq n$. Then,

$$
A_{n-1}\left[x_{1}\right]=K\left[x_{1}, \ldots, x_{n}\right]\left\langle\partial_{2}, \ldots, \partial_{n}\right\rangle .
$$

Definition 2.1. A multi-index $\alpha$ is an element of $\mathbb{N}^{n}$, say $\alpha=\left(\alpha_{1}, \ldots, \alpha_{n}\right)$. By $\partial^{\alpha}$, we mean the monomial $\partial_{1}{ }^{\alpha_{1}} \ldots \partial_{n}{ }^{\alpha_{n}}$. The order of this monomial is the length $|\alpha|$ of the multi-index $\alpha$; namely $|\alpha|=\alpha_{1}+\ldots+\alpha_{n}$. An element $d \in A_{n}(K)$ may be written uniquely in the form $d=\sum_{\alpha} q_{\alpha} \partial^{\alpha}$, where $q_{\alpha} \in K\left[x_{1}, \ldots, x_{n}\right]$. The order of $d$, denoted by $\operatorname{ord}(d)$, is the largest $|\alpha|$ for which $q_{\alpha} \neq 0$. We use the convention that the zero element has order $-\infty$. An example will suffice: the order of $x_{1}{ }^{3} \partial_{2}+x_{1}{ }^{7} x_{2} \partial_{1}{ }^{3} \partial_{2}{ }^{2}$ is equal to 5 .

We begin with some technical lemmas that will prepare for the proof of Theorem 2.8 .

Lemma 2.2. Let $S=\partial_{1}+\alpha_{2} \partial_{2}+\cdots+\alpha_{n} \partial_{n}+\gamma$ be an element in $A_{n}$, where $\alpha_{2}, \ldots, \alpha_{n}, \gamma \in K\left[x_{1}, \ldots, x_{n}\right]$. If $R \in A_{n-1}\left[x_{1}\right]$, then $[S, R] \in A_{n-1}\left[x_{1}\right]$. In particular, $\left[\partial_{1}, R\right] \in A_{n-1}\left[x_{1}\right]$.

Proof. This is a straightforward computation.

Lemma 2.3. (Division algorithm). Let $S=\partial_{1}+\alpha_{2} \partial_{2}+\cdots+\alpha_{n} \partial_{n}+\gamma$ be an element in $A_{n}$, where $\alpha_{2}, \ldots, \alpha_{n}, \gamma \in K\left[x_{1}, \ldots, x_{n}\right]$. Given $P \in A_{n}$, we have $P=Q S+R$, for some $Q \in A_{n}$ and $R \in A_{n-1}\left[x_{1}\right]$. Moreover, $R$ and $Q$ are uniquely determined.

Proof. We will first prove, by induction on $n$, that $\partial_{1}^{n}=Q S+R$, for some $Q \in A_{n}$ and $R \in A_{n-1}\left[x_{1}\right]$. 
Note that $\partial_{1}=1 S+R$, where $R=-\alpha_{2} \partial_{2}-\cdots-\alpha_{n} \partial_{n}-\gamma \in A_{n-1}\left[x_{1}\right]$. Suppose now that the result is true for $n$. Then

$$
\partial_{1}^{n+1}=\partial_{1} \partial_{1}^{n}=\partial_{1}(A S+B)=\partial_{1} A S+\partial_{1} B, A \in A_{n}, B \in A_{n-1}\left[x_{1}\right] .
$$

By lemma 2.2, we have

$$
\partial_{1}^{n+1}=\partial_{1} A S+B \partial_{1}+\widetilde{B}, \widetilde{B} \in A_{n-1}\left[x_{1}\right] .
$$

Since $\partial_{1}=S+R$

$$
\begin{aligned}
\partial_{1}^{n+1} & =\partial_{1} A S+B(S+R)+\widetilde{B} \\
& =\left(\partial_{1} A+B\right) S+B R+\widetilde{B} \\
& =Q^{\prime} S+R^{\prime}, \text { where } Q^{\prime} \in A_{n} \text { and } R^{\prime}=B R+\widetilde{B} \in A_{n-1}\left[x_{1}\right] .
\end{aligned}
$$

This completes the induction.

Now, if $P \in A_{n}$, we can write $P$ in the form $P=E_{n} \partial_{1}^{n}+\cdots+E_{1} \partial_{1}+E_{0}$, where $E_{i} \in A_{n-1}\left[x_{1}\right]$.

Thus, $P=E_{n}\left(H_{n} S+B_{n}\right)+\cdots+E_{1}\left(H_{1} S+B_{1}\right)+E_{0}$, where $H_{1}, \ldots, H_{n} \in A_{n}$ and $B_{1}, \ldots B_{n} \in A_{n-1}\left[x_{1}\right]$. Then,

$$
P=\left(E_{n} H_{n}+\cdots+E_{1} H_{1}\right) S+\underbrace{\left(E_{n} B_{n}+\cdots+E_{1} B_{1}+E_{0}\right)}_{\in A_{n-1}\left[x_{1}\right]}=Q S+R .
$$

We claim that $R$ is unique. In fact, let $R, R^{\prime} \in A_{n-1}\left[x_{1}\right]$ be such that $P=Q S+R=$ $Q^{\prime} S+R^{\prime}$. So, $R-R^{\prime}=a S$, for some $a \in A_{n}$. Writing $a=Q \partial_{1}+A$ and $S=\partial_{1}+B$, where $Q \in A_{n}, A, B \in A_{n-1}\left[x_{1}\right]$, we have

$$
a S=Q \partial_{1}^{2}+Q \partial_{1} B+A \partial_{1}+A B .
$$

Since $R, R^{\prime} \in A_{n-1}\left[x_{1}\right]$, so does $a S$. Hence, looking at $Q$ as a polynomial in $\partial_{1}$ with coefficients in $A_{n-1}\left[x_{1}\right]$ we conclude that $Q=0$. So, $a S=A \partial_{1}+A B$. In the same way, we conclude that $A=0$. Then $a=0, R=R^{\prime}$ and $Q=Q^{\prime}$.

LeMmA 2.4. Let $A_{n} S$ be a principal left ideal of $A_{n}$, where $S=\partial_{1}+\alpha_{2} \partial_{2}+\cdots+$ $\alpha_{n} \partial_{n}+\gamma \in A_{n}, \alpha_{2}, \ldots, \alpha_{n}$ and $\gamma \in K\left[x_{1}, \ldots, x_{n}\right]$. Then $A_{n} S$ is a maximal left ideal of $A_{n}$ if and only if $A_{n} S+A_{n} R=A_{n}$, for every $R \in A_{n-1}\left[x_{1}\right] \backslash\{0\}$.

Proof. $(\Rightarrow)$ If $R \in A_{n-1}\left[x_{1}\right] \backslash\{0\}$, then $R \notin A_{n} S$. So, $A_{n} S+A_{n} R=A_{n}$, because $A_{n} S$ is a maximal left ideal of $A_{n}$.

$(\Leftarrow)$ Of course, $A_{n} S$ is a maximal left ideal of $A_{n}$ if and only if $A_{n} S+A_{n} P=A_{n}$, for all $P \notin A_{n} S$. Now, for $P \notin A_{n} S$, by lemma 2.3, we have that $P=Q S+R$, for some $Q \in A_{n}$ and $R \in A_{n-1}\left[x_{1}\right], R \neq 0$. Thus, $A_{n} S+A_{n} P=A_{n} S+A_{n}(Q S+R)=$ $A_{n} S+A_{n} R=A_{n}$, by hypothesis.

LeMmA 2.5. Let $S=\partial_{1}+\alpha_{2} \partial_{2}+\cdots+\alpha_{n} \partial_{n}+\gamma$ be an element in $A_{n}$, where $\alpha_{2}, \ldots, \alpha_{n}, \gamma \in K\left[x_{1}, \ldots, x_{n}\right]$. Then, $A_{n}$ is a free $A_{n-1}\left[x_{1}\right]$-module with basis $\left\{1, S, S^{2}, \ldots\right\}$.

Proof. It is known that $A_{n}$ is a free $A_{n-1}\left[x_{1}\right]$-module with basis $\left\{1, \partial_{1}, \partial_{1}^{2}, \ldots\right\}$. Writing $\partial_{1}=S-R$ with $R=\alpha_{2} \partial_{2}+\cdots+\alpha_{n} \partial_{n}+\gamma \in A_{n-1}\left[x_{1}\right]$ and using lemma 2.2, we see that $A_{n}$ is generated by $\left\{1, S, S^{2}, \ldots\right\}$ over $A_{n-1}\left[x_{1}\right]$. 
Suppose that $r \geq 0$ and $B_{0}+B_{1} S+\cdots+B_{r} S^{r}=0$ with $B_{i} \in A_{n-1}\left[x_{1}\right]$ for every $i=0, \ldots, r$. Substituting $S$ by $\partial_{1}+R$ and using lemma 2.2 we have an expression $\widetilde{B}_{0}+\widetilde{B}_{1} \partial_{1}+\cdots+\widetilde{B}_{r} \partial_{1}^{r}=0$ with $\widetilde{B}_{i} \in A_{n-1}\left[x_{1}\right]$ for every $i=1, \ldots, r-1$ and $\widetilde{B}_{r}=B_{r}$. Therefore, $B_{r}=\widetilde{B}_{r}=0$. The proof follows by induction on $r$.

Lemma 2.6. Let $S \in A_{n}$ with $\operatorname{ord}(S)=1$ and $R \in A_{n-1}\left[x_{1}\right]$ with $\operatorname{ord}(R)>0$. Suppose that $\mu[S, R]=\eta R$ for some $\mu \in K\left[x_{1}, \ldots, x_{n}\right] \backslash\{0\}, \eta \in K\left[x_{1}, \ldots, x_{n}\right]$. Then, there exists $\widetilde{R} \in A_{n-1}\left[x_{1}\right]$, with $\operatorname{ord}(\widetilde{R})=\operatorname{ord}(R)$ and $\widetilde{\eta} \in K\left[x_{1}, \ldots, x_{n}\right]$ such that $[S, \widetilde{R}]=\widetilde{\eta} \widetilde{R}$.

Proof. We can write $R$ in the form

$$
\sum_{i_{2}+\ldots+i_{n}=0}^{N} P_{i_{2}, \ldots, i_{n}} \partial_{2}{ }^{i_{2}} \ldots \partial_{n}{ }^{i_{n}} \text {, where } P_{i_{2}, \ldots, i_{n}} \in K\left[x_{1}, \ldots, x_{n}\right] .
$$

Let $R=\alpha_{0} \widetilde{R}$ where $\alpha_{0} \in K\left[x_{1}, \ldots, x_{n}\right] \backslash\{0\}$ is the greatest common divisor of the elements $P_{i_{2}, \ldots, i_{n}}$. By hypothesis we have

$$
\mu\left[S, \alpha_{0} \widetilde{R}\right]=\eta \alpha_{0} \widetilde{R} .
$$

Since $\mu \in K\left[x_{1}, \ldots, x_{n}\right]$, by (1), $\mu$ divides $\eta \alpha_{0}$, say $\eta \alpha_{0}=\mu \zeta$ for some $\zeta \in$ $K\left[x_{1}, \ldots, x_{n}\right]$. Since $\mu \neq 0,\left[S, \alpha_{0} \widetilde{R}\right]=\zeta \widetilde{R}$.

But, $\left[S, \alpha_{0} \widetilde{R}\right]=\left[S, \alpha_{0}\right] \widetilde{R}+\alpha_{0}[S, \widetilde{R}]$. Then,

$$
\alpha_{0}[S, \widetilde{R}]=\underbrace{\left(\zeta-\left[S, \alpha_{0}\right]\right)}_{\lambda \in K\left[x_{1}, \ldots, x_{n}\right]} \widetilde{R}=\lambda \widetilde{R}, \text { where } \lambda \in K\left[x_{1}, \ldots, x_{n}\right] .
$$

It follows from (2), that $\alpha_{0}$ divides $\lambda$, say $\lambda=\tilde{\eta} \alpha_{0}$, for some $\tilde{\eta} \in K\left[x_{1}, \ldots, x_{n}\right]$. Since $\alpha_{0} \neq 0,[S, \widetilde{R}]=\widetilde{\eta} \widetilde{R}$.

LemMa 2.7. Let $S \in A_{n}$ with $\operatorname{ord}(S)=1$ and $P \in K\left[x_{1}, \ldots, x_{n}\right] \backslash K\left[x_{1}, \ldots, x_{n-1}\right]$. Suppose that $\mu[S, P]=\eta P$ for some $\mu \in K\left[x_{1}, \ldots, x_{n-1}\right] \backslash\{0\}$ and $\eta \in K\left[x_{1}, \ldots, x_{n}\right]$. Then, there exists $\widetilde{P} \in K\left[x_{1}, \ldots, x_{n}\right] \backslash K\left[x_{1}, \ldots, x_{n-1}\right]$ and $\widetilde{\eta} \in K\left[x_{1}, \ldots, x_{n}\right]$ such that $[S, \widetilde{P}]=\widetilde{\eta} \widetilde{P}$.

Proof. We can write $P$ in the form

$$
\sum_{i=0}^{N} P_{i} x_{n}^{i}, \text { where } P_{i} \in K\left[x_{1}, \ldots, x_{n-1}\right] \text { for every } i .
$$

Let $P=\alpha_{0} \widetilde{P}$ where $\alpha_{0} \in K\left[x_{1}, \ldots, x_{n-1}\right] \backslash\{0\}$ is the greatest common divisor of the elements $P_{i}$. By hypothesis we have

$$
\mu\left[S, \alpha_{0} \widetilde{P}\right]=\eta \alpha_{0} \widetilde{P} .
$$

Since $\mu \in K\left[x_{1}, \ldots, x_{n-1}\right]$, by (3), $\mu$ divides $\eta \alpha_{0}$, say $\eta \alpha_{0}=\mu \zeta$ for some $\zeta \in$ $K\left[x_{1}, \ldots, x_{n}\right]$. Since $\mu \neq 0,\left[S, \alpha_{0} \widetilde{P}\right]=\zeta \widetilde{P}$.

But, $\left[S, \alpha_{0} \widetilde{P}\right]=\left[S, \alpha_{0}\right] \widetilde{P}+\alpha_{0}[S, \widetilde{P}]$, hence $\alpha_{0}[S, \widetilde{P}]=\lambda \widetilde{P}$ where $\lambda:=\zeta-\left[S, \alpha_{0}\right] \in$ $K\left[x_{1}, \ldots, x_{n}\right]$. It follows that $\alpha_{0}$ divides $\lambda$, say $\lambda=\tilde{\eta} \alpha_{0}$, for some $\tilde{\eta} \in K\left[x_{1}, \ldots, x_{n}\right]$. Since $\alpha_{0} \neq 0,[S, \widetilde{P}]=\widetilde{\eta} \widetilde{P}$.

We can now state the main result of this section. 
THEOREM 2.8. Let $S=\partial_{1}+\alpha_{2} \partial_{2}+\cdots+\alpha_{n} \partial_{n}+\gamma$ be in $A_{n}$, where $\alpha_{2}, \ldots, \alpha_{n}$ and $\gamma \in K\left[x_{1}, \ldots, x_{n}\right]$.

(a) If $A_{n} S$ is a maximal left ideal of $A_{n}$, then $S$ has no Darboux operator in $A_{n-1}\left[x_{1}\right]$.

(b) Reciprocally, if $\alpha_{2} \in K\left[x_{1}, x_{2}\right], \ldots, \alpha_{n} \in K\left[x_{1}, x_{n}\right]$ and $S$ has no Darboux operator in $A_{n-1}\left[x_{1}\right]$, then $A_{n} S$ is a maximal left ideal of $A_{n}$.

(c) (Bratti and Takagi, [2, Theorem 2.2]) If $n=2$, then $A_{2} S$ is a maximal left ideal of $A_{2}$ if and only if $S$ has no Darboux operator in $A_{1}\left[x_{1}\right]$.

Proof. (a) Let $R \in A_{n-1}\left[x_{1}\right]$. Of course $R \notin A_{n} S$ and, since $A_{n} S$ is maximal, there exists $\lambda, \mu \in A_{n}$ such that $\lambda S+\mu R=1$. If $\operatorname{ord}_{\partial_{1}}(\lambda)=m$, then $\operatorname{ord}_{\partial_{1}}(\mu)=m+1$.

By lemma 2.5 we can write $\lambda$ and $\mu$ in the form:

$$
\begin{aligned}
\lambda & =B_{m} S^{m}+\cdots+B_{1} S+B_{0}, \\
\mu & =C_{m+1} S^{m+1}+\cdots+C_{1} S+C_{0},
\end{aligned}
$$

where $B_{i}, C_{j} \in A_{n-1}\left[x_{1}\right]$.

So,

$$
1=\lambda S+\mu R=\underbrace{\sum_{k=0}^{m} B_{k} S^{k+1}+\sum_{k=0}^{m+1} C_{k} S^{k} R}_{(*)}
$$

Suppose that $R$ is a Darboux operator for $S$ in $A_{n-1}\left[x_{1}\right]$, that is $R \in A_{n-1}\left[x_{1}\right] \backslash K$ and $[S, R]=\eta R$, for some $\eta \in K\left[x_{1}, \ldots, x_{n}\right]$. Then we have

$$
S^{m+1} R=R S^{m+1}+\left(\xi_{m} S^{m}+\xi_{m-1} S^{m-1}+\cdots+\xi_{1} S+\xi_{0}\right) R,
$$

with $\xi_{j} \in K\left[x_{1}, \ldots, x_{n}\right], 0 \leq j \leq m$. So, the coefficient of $S^{m+1}$ in $(*)$ is

$$
B_{m}+C_{m+1} R
$$

It follows from lemma 2.5 that

$$
B_{m}+C_{m+1} R=0
$$

Thus,

$$
\begin{aligned}
\lambda S+\mu R & =\sum_{k=0}^{m} B_{k} S^{k+1}+\sum_{k=0}^{m+1} C_{k} S^{k} R \\
& =\sum_{k=0}^{m-1} B_{k} S^{k+1}+\sum_{k=0}^{m} C_{k} S^{k} R+B_{m} S^{m+1}+C_{m+1} S^{m+1} R \\
& \stackrel{(5)}{=} \sum_{k=0}^{m-1} B_{k} S^{k+1}+\sum_{k=0}^{m} C_{k} S^{k} R-C_{m+1} R S^{m+1}+C_{m+1} S^{m+1} R \\
& =\sum_{k=0}^{m-1} B_{k} S^{k+1}+\sum_{k=0}^{m} C_{k} S^{k} R-C_{m+1}\left(R S^{m+1}-S^{m+1} R\right) .
\end{aligned}
$$


Using (4), we can rewrite (6) and obtain:

$$
1=\lambda S+\mu R=\sum_{k=0}^{m-1} B_{k} S^{k+1}+\sum_{k=0}^{m} \widetilde{C}_{k} S^{k} R
$$

for some $\widetilde{C}_{k} \in A_{n-1}\left[x_{1}\right]$.

This expression has the same form as $(*)$, but it involves only the powers $S^{i}$ with $i \leq m$.

Repeating the argument $m$ more times, we obtain

$$
1=\lambda S+\mu R=D_{0} R
$$

for some $D_{0} \in A_{n-1}\left[x_{1}\right]$. Then, $R$ is a unit of the Weyl algebra, hence $R \in K$, a contradiction.

(b) Let $R$ be in $A_{n-1}\left[x_{1}\right]$, such that $\operatorname{ord}(R)=N>0$. We can write $R$ in the form:

$$
R=\sum_{i_{2}+\ldots+i_{n}=0}^{N} P_{i_{2}, \ldots, i_{n}} \partial_{2}{ }^{i_{2}} \ldots \partial_{n}{ }^{i_{n}} \text {, where } P_{i_{2}, \ldots, i_{n}} \in K\left[x_{1}, \ldots, x_{n}\right] .
$$

Since ord $([S, R]) \leq \operatorname{ord}(S)+\operatorname{ord}(R)-1$, then $\operatorname{ord}([S, R]) \leq N$. Therefore we can also write $[S, R]$ in the form

$$
[S, R]=\sum_{i_{2}+\ldots+i_{n}=0}^{N} Q_{i_{2}, \ldots, i_{n}} \partial_{2}{ }^{i_{2}} \ldots \partial_{n}{ }^{i_{n}}, \text { where } Q_{i_{2}, \ldots, i_{n}} \in K\left[x_{1}, \ldots, x_{n}\right] .
$$

As $\operatorname{ord}(R)=N$, there exists $P_{i_{2_{0}}, \ldots, i_{n_{0}}} \neq 0$, such that

$$
i_{2_{0}}+\cdots+i_{n_{0}}=N
$$

By hypothesis and by lemma 2.6, we have that

$$
\widetilde{R}:=P_{i_{2_{0}}, \ldots, i_{n_{0}}}[S, R]-Q_{i_{2_{0}}, \ldots, i_{n_{0}}} R \neq 0 .
$$

Note that, from this equation, we have $0 \leq$ ord $\widetilde{R}$. Moreover, $\widetilde{R} \in A_{n} S+A_{n} R$ and the term of order $N$ involving $\partial_{2}{ }^{i_{2}} \ldots \partial_{n}{ }^{i_{n_{0}}}$ does not appear in $\widetilde{R}$. in $R$.

CLAIM 2.9. The multi-indices of maximal length that occur in $[S, R]$ already occur

Let's assume, for a while, that claim 2.9 is true. Then, $\widetilde{R}$ has one term less than $R$ of order $N$. If $\widetilde{R}$ has another term with order $N$, we can repeat the process and eliminate it too. Therefore, after a finite number of steps, we have a new $\widetilde{R} \in\left(A_{n} S+A_{n} R\right) \backslash\{0\}$, with $0 \leq \operatorname{ord}(\widetilde{R}) \leq N-1$. Proceeding in this way, we obtain

$$
\left(A_{n} S+A_{n} R\right) \cap\left(K\left[x_{1}, \ldots, x_{n}\right] \backslash\{0\}\right) \neq \emptyset .
$$

Let $P=\sum_{k=0}^{m} r_{k} x_{n}{ }^{k}$, where $r_{k} \in K\left[x_{1}, \ldots, x_{n-1}\right]$, be a polynomial contained in $\left(A_{n} S+A_{n} R\right) \cap\left(K\left[x_{1}, \ldots, x_{n}\right] \backslash\{0\}\right)$ with the least degree in $x_{n}$. If $m$ were strictly greater than 0 , then, by the Euclidean Algorithm (applied to $[S, P]$ and $P$ considered as elements in $\left.K\left(x_{1}, \ldots, x_{n-1}\right)\left[x_{n}\right]\right)$, there would exist $d \in K\left[x_{1}, \ldots, x_{n-1}\right] \backslash\{0\}$ such that 
$d[S, P]=\eta P+r$, for some $\eta, r \in K\left[x_{1}, \ldots, x_{n}\right]$ where $\operatorname{deg}_{x_{n}}(r)<\operatorname{deg}_{x_{n}}(P)$ or $r=0$. This would imply that $r=0$, by the choice of $P$, hence that $d[S, P]=\eta P$, which, by lemma 2.7, would lead to a contradiction with the hypothesis. So $m=0$ and $\left(A_{n} S+\right.$ $\left.A_{n} R\right) \cap\left(K\left[x_{1}, \ldots, x_{n-1}\right] \backslash\{0\}\right) \neq \emptyset$.

Proceeding in this way, we obtain

$$
\left(A_{n} S+A_{n} R\right) \cap\left(K\left[x_{1}\right] \backslash\{0\}\right) \neq \varnothing .
$$

Let $P=a_{l} x_{1}^{l}+\cdots+a_{0}$, with $a_{i} \in K$, be in $\left(A_{n} S+A_{n} R\right) \cap\left(K\left[x_{1}\right] \backslash\{0\}\right), a_{l} \neq 0$. If $l=0$, then $P \in K \backslash\{0\}$ and therefore $A_{n} S+A_{n} R=A_{n}$. If $l>0$, we have

$$
[S, P]=\partial_{1}(P)=l a_{l} x_{1}{ }^{l-1}+\cdots+a_{1} \in\left(A_{n} S+A_{n} R\right) \cap\left(K\left[x_{1}\right] \backslash\{0\}\right) .
$$

Repeating this process $l$ times, we have that $l ! a_{l} \in\left(A_{n} S+A_{n} R\right) \cap(K \backslash\{0\})$. Then $A_{n} S+A_{n} R=A_{n}$. By lemma 2.4, it follows that $A_{n} S$ is a maximal left ideal of $A_{n}$.

To finish the proof, we have to show claim 2.9.

Proof of claim 2.9: Let us suppose that

$$
S=d+\gamma \in A_{n},
$$

where $d=\partial_{1}+\alpha_{2} \partial_{2}+\cdots+\alpha_{n} \partial_{n}$ is a derivation of $K\left[x_{1}, \ldots, x_{n}\right]$ such that $\alpha_{i} \in$ $K\left[x_{1}, x_{i}\right], \gamma \in K\left[x_{1}, \ldots, x_{n}\right]$. Let $R=\sum_{i_{2}+\ldots+i_{n}=0}^{N} P_{i_{2}, \ldots, i_{n}} \partial_{2}{ }^{i_{2}} \ldots \partial_{n}{ }^{i_{n}}$, where $P_{i_{2}, \ldots, i_{n}} \in$ $K\left[x_{1}, \ldots, x_{n}\right]$.

Then,

$$
[S, R]=\left[S, \sum_{i_{2}+\ldots+i_{n}=0}^{N} P_{i_{2}, \ldots, i_{n}} \partial_{2}{ }^{i_{2}} \ldots \partial_{n}^{i_{n}}\right]=\sum_{i_{2}+\ldots+i_{n}=0}^{N}\left[S, P_{i_{2}, \ldots, i_{n}} \partial_{2}{ }^{i_{2}} \ldots \partial_{n}{ }^{i_{n}}\right] .
$$

Note that: $\left[\partial_{1}, P_{i_{2}, \ldots, i_{n}} \partial_{2}{ }^{i_{2}} \ldots \partial_{n}{ }^{i_{n}}\right]=\partial_{1}\left(P_{i_{2}, \ldots, i_{n}}\right) \partial_{2}{ }^{i_{2}} \ldots \partial_{n}{ }^{i_{n}}$, and

$$
\begin{aligned}
& {\left[\alpha_{2} \partial_{2}, P_{i_{2}, \ldots, i_{n}} \partial_{2}{ }^{i_{2}} \ldots \partial_{n}{ }^{i_{n}}\right]=\alpha_{2} P_{i_{2}, \ldots, i_{n}} \partial_{2}{ }^{i_{2}+1} \partial_{3}{ }^{i_{3}} \ldots \partial_{n}{ }^{i_{n}}+\alpha_{2} \partial_{2}\left(P_{i_{2}, \ldots, i_{n}}\right) \partial_{2}{ }^{i_{2}} \ldots \partial_{n}{ }^{i_{n}} } \\
&-P_{i_{2}, \ldots, i_{n}} \partial_{2}{ }^{i_{2}} \ldots \partial_{n}{ }^{i_{n}} \alpha_{2} \partial_{2} \\
&= \alpha_{2} P_{i_{2}, \ldots, i_{n}} \partial_{2}{ }^{i_{2}+1} \partial_{3}{ }^{i_{3}} \ldots \partial_{n}{ }^{i_{n}}+\alpha_{2} \partial_{2}\left(P_{i_{2}, \ldots, i_{n}}\right) \partial_{2}{ }^{i_{2}} \ldots \partial_{n}{ }^{i_{n}}-P_{i_{2}, \ldots, i_{n}} \partial_{2}{ }^{i_{2}} \alpha_{2} \partial_{2} \partial_{3}{ }^{i_{3}} \ldots \partial_{n}{ }^{i_{n}} \\
&= \alpha_{2} P_{i_{2}, \ldots, i_{n}} \partial_{2}{ }^{i_{2}+1} \partial_{3}{ }^{i_{3}} \ldots \partial_{n}{ }^{i_{n}}+\alpha_{2} \partial_{2}\left(P_{i_{2}, \ldots, i_{n}}\right) \partial_{2}{ }^{i_{2}} \ldots \partial_{n}{ }^{i_{n}}-P_{i_{2}, \ldots, i_{n}}\left(\alpha_{2} \partial_{2}{ }^{i_{2}+1} \partial_{3}{ }^{i_{3}} \ldots \partial_{n}{ }^{i_{n}}\right. \\
&\left.+i_{2} \partial_{2}\left(\alpha_{2}\right) \partial_{2}{ }^{i_{2}} \ldots \partial_{n}{ }^{i_{n}}+\text { terms with lower order }\right) \\
&=\left(\alpha_{2} \partial_{2}\left(P_{i_{2}, \ldots, i_{n}}\right)-i_{2} P_{i_{2}, \ldots, i_{n}} \partial_{2}\left(\alpha_{2}\right)\right) \partial_{2}{ }^{i_{2}} \ldots \partial_{n}{ }^{i_{n}}+\text { terms with lower order }
\end{aligned}
$$

Hence, the terms with order $N$ in $\sum_{i_{2}+\ldots+i_{n}=0}^{N}\left[\alpha_{2} \partial_{2}, P_{i_{2}, \ldots, i_{n}} \partial_{2}{ }^{i_{2}} \ldots \partial_{n}{ }^{i_{n}}\right]$ are:

$$
\sum_{i_{2}+\ldots i_{n}=N}\left(\alpha_{2} \partial_{2}\left(P_{i_{2}, \ldots, i_{n}}\right)-i_{2} P_{i_{2}, \ldots, i_{n}} \partial_{2}\left(\alpha_{2}\right)\right) \partial_{2}{ }^{i_{2}} \ldots \partial_{n}{ }^{i_{n}} .
$$

Similarly, the terms with order $N$ in $\sum_{i_{2}+\ldots+i_{n}=0}^{N}\left[\alpha_{j} \partial_{j}, P_{i_{2}, \ldots, i_{n}} \partial_{2}{ }^{i_{2}} \ldots \partial_{n}{ }^{i_{n}}\right]$, where $j=$ $2, \ldots, n$, are:

$$
\sum_{i_{2}+\ldots i_{n}=N}\left(\alpha_{j} \partial_{j}\left(P_{i_{2}, \ldots, i_{n}}\right)-i_{j} P_{i_{2}, \ldots, i_{n}} \partial_{j}\left(\alpha_{j}\right)\right) \partial_{2}{ }^{i_{2}} \ldots \partial_{n}{ }^{i_{n}}
$$


Note that $\operatorname{ord}([\gamma, R]) \leq N-1$. Then the terms with order $N$ in $[S, R]$ are:

$$
\sum_{i_{2}+\ldots+i_{n}=N}\left(d\left(P_{i_{2}, \ldots, i_{n}}\right)-\sum_{j=2}^{n} i_{j} P_{i_{2}, \ldots, i_{n}} \partial_{j}\left(\alpha_{j}\right)\right) \partial_{2}{ }^{i_{2}} \ldots \partial_{n}{ }^{i_{n}} .
$$

Now, observe that if $P_{i_{2}, \ldots, i_{n}}$ is the coefficient of $\partial_{2}{ }^{i_{2}} \ldots \partial_{n}{ }^{i_{n}}$ in $R$, with $i_{2}+\ldots+i_{n}=N$, then the corresponding coefficient in $[S, R]$ is:

$$
Q_{i_{2}, \ldots, i_{n}}:=d\left(P_{i_{2}, \ldots, i_{n}}\right)-\sum_{j=2}^{n} i_{j} P_{i_{2}, \ldots, i_{n}} \partial_{j}\left(\alpha_{j}\right) .
$$

Therefore, if $P_{i_{2}, \ldots, i_{n}}=0$, then $Q_{i_{2}, \ldots, i_{n}}=0$ and the coefficient of $\partial_{2}{ }^{i_{2}} \ldots \partial_{n}{ }^{i_{n}}$ in $[S, R]$ is zero.

3. $\boldsymbol{d}$-simplicity of the ring $K\left[x_{1}, \ldots, x_{n}\right]$. In this section we study the $d$-simplicity of the ring $K\left[x_{1}, \ldots, x_{n}\right]$. Evidently, if $K\left[x_{1}, \ldots, x_{n}\right]$ is $d$-simple, there is no non-trivial principal $d$-ideal (equivalently, there is no Darboux polynomial). If $d\left(x_{1}\right)=1$, the converse is true when $n=2$ but is false already when $n=3$. Indeed, if $K$ is a formally real field (for example if $K=\mathbb{R}$ ), Goodearl and Warfield observed in ([6, p. 61]) that in $K\left[x_{2}, x_{3}\right]$, the derivation $\delta:=\left(x_{2}+x_{3}\right) \partial_{2}+\left(x_{2}^{2}+x_{3}^{2}\right) \partial_{3}$ has no Darboux polynomial even though $\left(x_{2}, x_{3}\right)$ is a (unique) $\delta$-ideal; then, by a rather straightforward computation, one can see that in $K\left[x_{1}, x_{2}, x_{3}\right]$, the derivation $d:=\partial_{1}+\left(x_{2}+x_{3}\right) \partial_{2}+$ $\left(x_{2}^{2}+x_{3}^{2}\right) \partial_{3}$ has no Darboux polynomial even though $\left(x_{2}, x_{3}\right) K\left[x_{1}, x_{2}, x_{3}\right]$ is a (unique) $d$-ideal. Our next theorem gives a rather general situation where the converse is true; it points out that the peculiarity of the above example would not have occurred if the coefficient of $\partial_{2}$ had been an element of $K\left[x_{1}, x_{2}\right.$ ]. It generalizes [9, Proposition 2.1].

THEOREM 3.1. Let $d=\partial_{1}+\alpha_{2} \partial_{2}+\cdots+\alpha_{n} \partial_{n}$ be a derivation of $K\left[x_{1}, \ldots, x_{n}\right]$ where $\alpha_{i} \in K\left[x_{1}, \ldots, x_{i}\right]$ for every $i=2, \ldots, n$. Then the following statements are equivalent:

(i) $K\left[x_{1}, \ldots, x_{n}\right]$ is $d$-simple.

(ii) d has no Darboux polynomial.

Proof. It is enough to show that (ii) $\Rightarrow$ (i). Suppose that $K\left[x_{1}, \ldots, x_{n}\right]$ is not $d$-simple. Let $I$ be a proper non-zero $d$-ideal of $K\left[x_{1}, \ldots, x_{n}\right]$. Let $P=\sum_{k=0}^{l} r_{k} x_{n}{ }^{k}$, where $r_{k} \in K\left[x_{1}, \ldots, x_{n-1}\right]$, be a non-zero polynomial contained in $I$ with the least degree in $x_{n}$.

Suppose that $l>0$. By the usual Euclidean Algorithm (applied to $d(P)$ and $P$ considered as elements in $\left.K\left(x_{1}, \ldots, x_{n-1}\right)\left[x_{n}\right]\right)$, there exists $g \in K\left[x_{1}, \ldots, x_{n-1}\right] \backslash\{0\}$ such that $g d(P)=h P+r$, for some $h, r \in K\left[x_{1}, \ldots, x_{n}\right]$, where $\operatorname{deg}_{x_{n}}(r)<\operatorname{deg}_{x_{n}}(P)$ or $r=0$. This implies that $r=0$, by the choice of $P$. Thus, $g d(P)=h P$. Since $d(P)=[d, P]$, then by lemma 2.7 , there exist $\widetilde{h} \in K\left[x_{1}, \ldots, x_{n}\right]$ and $\widetilde{P} \in K\left[x_{1}, \ldots, x_{n}\right] \backslash$ $K\left[x_{1}, \ldots, x_{n-1}\right]$ such that $[d, \widetilde{P}]=\widetilde{h} \widetilde{P}$. As $d(\widetilde{P})=[d, \widetilde{P}], \widetilde{P}$ is a Darboux polynomial of $d$, a contradiction to the hypothesis.

Thus $l=0$ and $I \cap K\left[x_{1}, \ldots, x_{n-1}\right] \neq(0)$. Note that $\left.d\right|_{K\left[x_{1}, \ldots, x_{n-1}\right]}\left(K\left[x_{1}, \ldots\right.\right.$, $\left.\left.x_{n-1}\right]\right) \subseteq K\left[x_{1}, \ldots, x_{n-1}\right]$, since $\alpha_{i} \in K\left[x_{1}, \ldots, x_{i}\right]$. Then we can repeat the argument.

Going on this way, we obtain that $I \cap K\left[x_{1}\right] \neq(0)$. But this is impossible since $d$ restricted to $K\left[x_{1}\right]$ is $\partial_{1}$ and $\partial_{1}$ is a simple derivation of $K\left[x_{1}\right]$.

COROLlary 3.2. Let $d=\partial_{1}+\alpha_{2} \partial_{2}+\cdots+\alpha_{n} \partial_{n}$ be a derivation of $K\left[x_{1}, \ldots, x_{n}\right]$ where $\alpha_{i} \in K\left[x_{1}, \ldots, x_{i}\right]$ for every $i=2, \ldots, n$. Then, $K\left[x_{1}, \ldots, x_{n}\right] i$ is $d$-simple if and only if no prime ideal of height one is a d-ideal. 
Proof. By Theorem 3.1, $K\left[x_{1}, \ldots, x_{n}\right]$ is $d$-simple if and only if no non-zero proper principal ideal is a $d$-ideal. But if an ideal $I$ is a $d$-ideal, then every minimal prime of $I$ is also a $d$-ideal. By Krull's Principal Ideal Theorem, every minimal prime ideal of a principal ideal has height one.

COROLlaRY 3.3. Let $d=\partial_{1}+\alpha_{2} \partial_{2}+\cdots+\alpha_{n} \partial_{n}$ be a derivation of $K\left[x_{1}, \ldots, x_{n}\right]$, with $\alpha_{i} \in K\left[x_{1}, \ldots, x_{n}\right]$ for every $i=2, \ldots, n$. Suppose that there exists $\gamma \in$ $K\left[x_{1}, \ldots, x_{n}\right]$ such that $A_{n}(d+\gamma)$ is a maximal left ideal of $A_{n}$. Then,

(a) $d$ has no Darboux polynomial.

(b) $d$ is a simple derivation if $\alpha_{i} \in K\left[x_{1}, \ldots, x_{i}\right]$, for every $i=2, \ldots, n$.

Proof. (a): Suppose that $A_{n}(d+\gamma)$ is a maximal left ideal of $A_{n}$. By Theorem 2.8(a), we have

$$
[d+\gamma, R] \notin K\left[x_{1}, \ldots, x_{n}\right] R, \forall R \in A_{n-1}\left[x_{1}\right] \backslash K .
$$

So, in particular

$$
[d+\gamma, P] \notin K\left[x_{1}, \ldots, x_{n}\right] P, \forall P \in K\left[x_{1}, \ldots, x_{n}\right] \backslash K .
$$

Since $[d+\gamma, P]=[d, P]=d(P), d$ has no Darboux polynomial.

(b): It follows from item (a) and Theorem 3.1.

Examples of simple derivations of the polynomial ring $K\left[x_{1}, \ldots, x_{n}\right]$ are not easy to find. A family of linear simple derivations was discovered by Coutinho in [3] (generalizing an example of Stafford) and is based on a result of Shamsuddin (see [10]). Families of simple quadratic derivations of $K\left[x_{1}, x_{2}\right]$ were found by Maciejewski, Moulin-Ollagnier and Nowicki in [9].

EXAmPLE 3.4. Let $K \supsetneqq \mathbb{Q}$ be a field and $S$ be the element in $A_{2}(K)$, given by

$$
S=\partial_{1}+\left(x_{1} x_{2}+\lambda x_{2}{ }^{2}+1\right) \partial_{2}+\lambda \mu x_{2}, \lambda \in K \backslash \mathbb{Q} \text { and } \mu \notin \mathbb{Z}
$$

Stafford proved in [11, Proposition 2.2] that $A_{2} S$ is a maximal left ideal of $A_{2}(K)$ (actually, our operator is obtained from Stafford's after a transposition and a change of indices).

Consider the derivation $d=\partial_{1}+\left(x_{1} x_{2}+\lambda x_{2}^{2}+1\right) \partial_{2}$ of $K\left[x_{1}, x_{2}\right]$ extracted from S. By Corollary 3.3, we have that $K\left[x_{1}, x_{2}\right]$ is $d$-simple. Note that in this case, we get an example where $K\left[x_{1}, x_{2}\right]$ is $d$-simple and $d$ is not a Shamsuddin derivation.

The following lemma will be used in the proof of the next theorem.

If $\mathcal{A}$ denotes a commutative domain, let $q f(\mathcal{A})$ denote its field of quotients and let $\mathcal{A}^{*}$ denote its group of units.

LEMMA 3.5. Let $\mathcal{A}$ be a $K$-algebra which is a factorial domain and $d$ a $K$-derivation of $\mathcal{A}$. Suppose that $\mathcal{A}$ has no non-zero proper principal d-ideals. Given $f, g \in \mathcal{A}$ consider the following differential equation:

$$
d(u)+f u=g .
$$

(a) If $u \in q f(\mathcal{A})$ is a solution of (8), then $u \in \mathcal{A}$

(b) If $g=0$ and $u \in \mathcal{A}$ is a nontrivial solution of (8), then $u \in \mathcal{A}^{*}$. In particular, if $\mathcal{A}^{*}=K^{*}$ and $f \neq 0$, then equation (8) has only the trivial solution. 
Proof. (a) Suppose that $u=\frac{p}{q} \in q f(\mathcal{A})$, with $\operatorname{gcd}(p, q)=1$, is such that

$$
d\left(\frac{p}{q}\right)+f \frac{p}{q}=g
$$

Then

$$
q(d(p)+f p-g q)=p d(q)
$$

As $\operatorname{gcd}(p, q)=1$, there exists $r \in \mathcal{A}$ such that

$$
\left\{\begin{array}{l}
d(p)+f p-g q=r p \\
d(q)=r q
\end{array}\right.
$$

Therefore, $(q) \subset \mathcal{A}$ is a $d$-ideal of $\mathcal{A}$. Then $(q)=(0)$ or $(q)=(1)$. As $q \neq 0$, it follows that $q \in \mathcal{A}^{*}$. Hence $u=\frac{p}{q} \in \mathcal{A}$.

(b) Let $p \in \mathcal{A}$ be a solution of $d(u)+f u=0$. Then $(p)$ is a $d$-ideal and $p=0$ or $p \in \mathcal{A}^{*}$. If $\mathcal{A}^{*}=K^{*}$, as $d$ is a $K$-derivation, we have that $f p=0$. Then $p=0$.

A characterization of the $d$-simplicity of the $\operatorname{ring} K\left[x_{1}, x_{2}\right]$, where $d$ is a Shamsuddin derivation, is given in [10] in terms of the existence of a polynomial solution of a certain ODE. The following theorem generalizes this result for an arbitrary number of variables.

THEOREM 3.6. Let $d=\partial_{1}+\alpha_{2} \partial_{2}+\cdots+\alpha_{n} \partial_{n}$ be a Shamsuddin derivation of $K\left[x_{1}, \ldots, x_{n}\right]$, where $\alpha_{i}\left(x_{1}, x_{i}\right)=a_{i}\left(x_{1}\right) x_{i}+b_{i}\left(x_{1}\right) \in K\left[x_{1}, x_{i}\right], 2 \leq i \leq n$. Suppose that $a_{i} \neq a_{j}$, for $2 \leq i<j \leq n$. Then the following statements are equivalent:

(i) $K\left[x_{1}, \ldots, x_{n}\right]$ is $d$-simple.

(ii) $\partial_{1}(v) \neq a_{i} \cdot v+b_{i}$, for every $v \in K\left(x_{1}\right)$, for all $i=2, \ldots, n$.

(iii) $\partial_{1}(v) \neq a_{i} \cdot v+b_{i}$, for every $v \in K\left[x_{1}\right]$, for all $i=2, \ldots, n$.

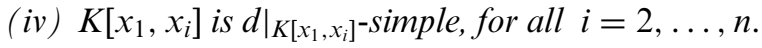

Proof. (ii) $\Leftrightarrow$ (iii) is given by Lemma 3.9 applied with $\mathcal{A}=K\left[x_{1}\right]$ and $d=\partial_{1}$.

(iii) $\Leftrightarrow$ (iv) is given by Shamsuddin's Theorem ([3, Proposition 3.2]).

(i) $\Rightarrow$ (iv). If $I$ is a non-zero proper $\left.d\right|_{K\left[x_{1}, x_{i}\right]}$-ideal of $K\left[x_{1}, x_{i}\right]$, then $I K\left[x_{1}, \ldots, x_{n}\right]$ is a non-zero proper $d$-ideal of $K\left[x_{1}, \ldots, x_{n}\right]$.

(ii) $\Rightarrow$ (i). Let $I$ be a non-zero $d$-ideal of $K\left[x_{1}, \ldots, x_{n}\right]$ and let $P \in I, P \neq 0$. We can suppose that $P$ is not a constant. We write $P$ in the form:

$$
P=\sum_{i_{2}+\cdots+i_{n}=0}^{N} P_{i_{2}, \ldots, i_{n}} x_{2}{ }^{i_{2}} \cdots x_{n}^{i_{n}}, \text { where } P_{i_{2}, \ldots, i_{n}} \in K\left[x_{1}\right]
$$

Then, a simple calculation gives the following expression for $d(P)$ :

$$
\begin{aligned}
d(P)= & \sum_{i_{2}+\cdots+i_{n}=0}^{N}\left\{\left(\partial_{1}\left(P_{i_{2}, \ldots, i_{n}}\right)+i_{2} P_{i_{2}, \ldots, i_{n}} a_{2}+\cdots+i_{n} P_{i_{2}, \ldots, i_{n}} a_{n}\right) x_{2}{ }^{i_{2}} \cdots x_{n}^{i_{n}}+\right. \\
& \left.+\left(i_{2} P_{i_{2}, \ldots, i_{n}} b_{2}\right) x_{2}{ }^{i_{2}-1} \cdots x_{n}{ }^{i_{n}}+\cdots+\left(i_{n} P_{i_{2}, \ldots, i_{n}} b_{n}\right) x_{2}{ }^{i_{2}} \cdots x_{n}{ }^{i_{n}-1}\right\}
\end{aligned}
$$

Let us choose $P \in I$ such that $N$ is minimum. If $N=0$ then $P \in K\left[x_{1}\right] \backslash K$ and we are done; indeed, if degree $P=r$, then $d^{(r)}(P)$ is a unit that belongs to $I$. 
Suppose that $N>0$. So, there exists $P_{j_{2}, \ldots, j_{n}} \neq 0$ for some $j_{2}+\cdots+j_{n}=N$. Without loss of generality we may suppose that $j_{2}>0$. Note that

$$
\rho:=\partial_{1}\left(P_{j_{2}, \ldots, j_{n}}\right)+j_{2} P_{j_{2}, \ldots, j_{n}} a_{2}+\cdots+j_{n} P_{j_{2}, \ldots, j_{n}} a_{n}
$$

is the coefficient of the monomial $x_{2}{ }^{j_{2}} \cdots x_{n}{ }^{j_{n}}$ in $d(P)$. We consider

$$
P_{1}:=P_{j_{2}, \ldots, j_{n}} d(P)-\rho P \in I
$$

Evidently, $P_{1}$ has no term in $x_{2}^{j_{2}} \cdots x_{n}^{j_{n}}$, while the coefficient of the term $x_{2}^{j_{2}-1} \cdots x_{n}^{j_{n}}$, say $\zeta_{j_{2}-1, \ldots, j_{n}} \in K\left[x_{1}\right]$, is the following:

$$
\begin{aligned}
\zeta_{j_{2}-1, \ldots, j_{n}}= & P_{j_{2}, \ldots, j_{n}}^{2}\left(\partial_{1}\left(\frac{P_{j_{2}-1, \ldots, j_{n}}}{P_{j_{2}, \ldots, j_{n}}}\right)-a_{2} \frac{P_{j_{2}-1, \ldots, j_{n}}}{P_{j_{2}, \ldots, j_{n}}}+j_{2} b_{2}\right. \\
& \left.+\left(j_{3}+1\right) b_{3} \frac{P_{j_{2}-1, j_{3}+1, j_{4}, \ldots, j_{n}}}{P_{j_{2}, \ldots, j_{n}}}+\cdots+\left(j_{n}+1\right) b_{n} \frac{P_{j_{2}-1, j_{3}, \ldots, j_{n-1}, j_{n}+1}}{P_{j_{2}, \ldots, j_{n}}}\right) .
\end{aligned}
$$

We will analyze two cases.

FIRST CASE: If $P_{j_{2}-1, j_{3}+1, j_{4}, \ldots, j_{n}}=\cdots=P_{j_{2}-1, j_{3}, \ldots, j_{n-1}, j_{n}+1}=0$.

We claim that $P_{1} \neq 0$. Indeed, in this case, equation (10) simplifies and the coefficient of the term $x_{2}^{j_{2}-1} \cdots x_{n}^{j_{n}}$ is

$$
\zeta_{j_{2}-1, \ldots, j_{n}}=P_{j_{2}, \ldots, j_{n}}^{2} j_{2}\left(\partial_{1}\left(\frac{P_{j_{2}-1, \ldots, j_{n}}}{j_{2} P_{j_{2}, \ldots, j_{n}}}\right)-a_{2} \frac{P_{j_{2}-1, \ldots, j_{n}}}{j_{2} P_{j_{2}, \ldots, j_{n}}}+b_{2}\right)
$$

which is non-zero by hypothesis (ii).

Therefore, the ideal $I$ contains a non-zero element $P_{1}$ without the term $x_{2}{ }^{j_{2}} \cdots x_{n}{ }^{j_{n}}$.

SECOND CASE: If $P_{j_{2}-1, \ldots, j_{k}+1, \ldots, j_{n}} \neq 0$, for some $k, 3 \leq k \leq n$.

Note that

$$
\begin{aligned}
\psi_{j_{2}-1, \ldots, j_{k}+1, \ldots, j_{n}}: & =\partial_{1}\left(P_{j_{2}-1+, \ldots, j_{k}+1, \ldots, j_{n}}\right)+\left(j_{2}-1\right) P_{j_{2}-1, \ldots, j_{k}+1, \ldots, j_{n}} a_{2} \\
& +j_{3} P_{j_{2}-1, \ldots, j_{k}+1, \ldots, j_{n}} a_{3}+\cdots+\left(j_{k}+1\right) P_{j_{2}-1, \ldots, j_{k}+1, \ldots, j_{n}} a_{k}+\cdots \\
& +j_{n} P_{j_{2}-1, \ldots, j_{k}+1, \ldots, j_{n}} a_{n}
\end{aligned}
$$

is the coefficient of the monomial $x_{2}^{j_{2}-1} \cdots x_{k}^{j_{k}+1} \cdots x_{n}^{j_{n}}$ in $d(P)$.

Consider

$$
P_{2}:=P_{j_{2}-1, \ldots, j_{k}+1, \ldots, j_{n}} d(P)-\psi_{j_{2}-1, \ldots, j_{k}+1, \ldots, j_{n}} P \in I
$$

Evidently, $P_{2}$ has no term in $x_{2}^{j_{2}-1} \cdots x_{k}^{j_{k}+1} \cdots x_{n}^{j_{n}}$, while the coefficient of the term $x_{2}^{j_{2}} \cdots x_{n}^{j_{n}}$, say $\vartheta_{j_{2}, \ldots, j_{n}} \in K\left[x_{1}\right]$, is the following:

$$
\vartheta_{j_{2}, \ldots, j_{n}}=P_{j_{2}-1, \ldots, j_{k}+1, \ldots, j_{n}}^{2}\left(\partial_{1}\left(\frac{P_{j_{2}, \ldots, j_{n}}}{P_{j_{2}-1, \ldots, j_{k}+1, \ldots, j_{n}}}\right)+\left(a_{2}-a_{k}\right) \frac{P_{j_{2}, \ldots, j_{n}}}{P_{j_{2}-1, \ldots, j_{k}+1, \ldots, j_{n}}}\right) .
$$

Hence, from Lemma 3.5 and from the fact that $a_{2} \neq a_{k}$, we obtain that $\vartheta_{j_{2}, \ldots, j_{n}} \neq 0$. Then, the coefficient of $x_{2}^{j_{2}} \cdots x_{n}^{j_{n}}$ in $P_{2}$ is nonzero, while its coefficient in $x_{2}^{j_{n}-1} \cdots x_{k}^{j_{k}+1} \ldots x_{n}^{j_{n}}$ is zero. Repeating this argument for every $k=3, \ldots, n$ such that $P_{j_{2}-1, \ldots, j_{k}+1, \ldots, j_{n}} \neq 0$, we obtain a nonzero element $\tilde{P} \in I$ such that its coefficient of 
$x_{2}^{j_{2}} \cdots x_{n}^{j_{n}}$ is non-zero while all the coefficients of $x_{2}^{j_{2}-1} \cdots x_{k}^{j_{k}+1} \cdots x_{n}^{j_{n}}$, for $3 \leq k \leq n$, are zero. We are back to the first case.

In any case, we get a nonzero element in $I$ that does not involve the monomial $x_{2}^{j_{2}} \cdots x_{n}^{j_{n}}$. Iterating this argument, we have that $I$ contains a nonzero element $Q$ of the form $Q=\sum_{i_{2}+\cdots+i_{n}=0}^{N-1} Q_{i_{2}, \ldots, i_{n}} x_{2}^{i_{2}} \cdots x_{n}^{i_{n}}$. This is a contradiction with the minimality of $N$.

The next example shows that the hypothesis $a_{i} \neq a_{j}$, for $i \neq j$, in Theorem 3.6 cannot be dropped in general.

EXAMPLE 3.7. Let $d=\partial_{1}+\left(x_{1} x_{2}+1\right) \partial_{2}+\left(x_{1} x_{3}+1\right) \partial_{3}$ be a derivation of $K\left[x_{1}, x_{2}, x_{3}\right]$. Let $I=\left(x_{2}-x_{3}\right) K\left[x_{1}, x_{2}, x_{3}\right]$. Then $d\left(x_{2}-x_{3}\right)=x_{1}\left(x_{2}-x_{3}\right)$ and $I$ is a non-zero, proper $d$-ideal. Therefore, $d$ is not a simple derivation of $K\left[x_{1}, x_{2}, x_{3}\right]$, even though $K\left[x_{1}, x_{i}\right]$ is $\left.d\right|_{K\left[x_{1}, x_{i}\right]}$-simple for $i=2,3$.

We will now use our theorem 3.6 to recover [3, Theorem 3.3]. Coutinho considers, for $2 \leq i \leq n$, non-zero polynomials $a_{i}, b_{i} \in K\left[x_{1}\right]$ such that:

(1) $\frac{a_{i}}{a_{j}} \notin \mathbb{Q}$ whenever $2 \leq i<j \leq n$ and

(2) $\operatorname{deg}\left(a_{i}\right)>\operatorname{deg}\left(b_{i}\right)$ for $i=2, \ldots, n$.

He shows that $d=\partial_{1}+\sum_{i=2}^{n}\left(x_{i} a_{i}+b_{i}\right) \partial_{i}$ is a simple derivation of the ring $K\left[x_{1}, \ldots, x_{n}\right]$. One advantage of our approach is that we can weaken the conditions on the polynomials $a_{2}, \ldots, a_{n}$.

EXAMPLE 3.8. Consider, for $2 \leq i \leq n$, non-zero polynomials $a_{i}, b_{i} \in K\left[x_{1}\right]$ such that $\operatorname{deg}\left(a_{i}\right)>\operatorname{deg}\left(b_{i}\right)$ and $a_{i} \neq a_{j}$ for $2 \leq i<j \leq n$. Then,

$$
d=\partial_{1}+\sum_{i=2}^{n}\left(x_{i} a_{i}+b_{i}\right) \partial_{i}
$$

is a simple derivation of the $\operatorname{ring} K\left[x_{1}, \ldots, x_{n}\right]$.

In fact, we must check if

$$
\partial_{1}(v) \neq a_{i} \cdot v+b_{i},
$$

for every $v \in K\left[x_{1}\right]$ and for every $i=2, \ldots, n$.

Observe that if $v \in K\left[x_{1}\right]$ is a solution of $\partial_{1}(v)=a_{i} \cdot v+b_{i}$, then,

$$
\underbrace{\partial_{1}(v)}_{\operatorname{deg}(v)-1}-\underbrace{a_{i} \cdot v}_{\operatorname{deg}\left(a_{i}\right)+\operatorname{deg}(v)}=\underbrace{b_{i}}_{\operatorname{deg}\left(b_{i}\right)}, i=2, \ldots, n .
$$

Since $\operatorname{deg}\left(a_{i}\right)>\operatorname{deg}\left(b_{i}\right), i=2, \ldots, n$, none of these equations has a solution in $K\left[x_{1}\right]$.

By theorem 3.6, it follows that $K\left[x_{1}, \ldots, x_{n}\right]$ is $d$-simple.

Now we give another new family of simple derivations of the ring $K\left[x_{1}, \ldots, x_{n}\right]$. They are Shamsuddin derivations.

EXAMPLE 3.9. For $2 \leq i \leq n$, let $f_{i}, g_{i}$ be monic polynomials in $K\left[x_{1}\right]$ such that $\operatorname{deg}\left(f_{i}\right)=\operatorname{deg}\left(g_{i}\right)$, and $f_{i} \neq f_{j}, 2 \leq i<j \leq n$. Then the following derivation

$$
d=\partial_{1}+\left(x_{1}^{2} g_{2}+x_{1} f_{2} x_{2}\right) \partial_{2}+\cdots+\left(x_{1}^{2} g_{n}+x_{1} f_{n} x_{n}\right) \partial_{n}
$$

is a simple derivation of the ring $K\left[x_{1}, \ldots, x_{n}\right]$. 
In fact, we must check if

$$
\partial_{1}(v) \neq x_{1} f_{i} v+x_{1}^{2} g_{i}
$$

for every $v \in K\left[x_{1}\right]$ and for every $i=2, \ldots, n$. Let $k_{i}:=\operatorname{deg}\left(f_{i}\right)=\operatorname{deg}\left(g_{i}\right)$. If $v \in K\left[x_{1}\right]$ is such that $\partial_{1}(v)=x_{1} f_{i} v+x_{1}^{2} g_{i}$, then

$$
\underbrace{\partial_{1}(v)}_{\operatorname{deg}(v)-1}-\underbrace{x_{1} f_{i} v}_{\operatorname{deg}(v)+k_{i}+1}=\underbrace{x_{1}{ }^{2} g_{i}}_{k_{i}+2} .
$$

Hence $\operatorname{deg}(v)=1$.

We can write $f_{i}, g_{i}$ and $v$ in the form:

$$
\begin{aligned}
f_{i} & =x_{1}{ }^{k_{i}}+{ }_{i} f_{k_{i}-1} x_{1}{ }^{k_{i}-1}+\cdots+{ }_{i} f_{0} \\
g_{i} & =x_{1}{ }^{k_{i}}+{ }_{i} g_{k_{i}-1} x_{1}{ }^{k_{i}-1}+\cdots+{ }_{i} g_{0} \\
v & =c x_{1}+e
\end{aligned}
$$

with $f_{j},{ }_{i} g_{j} \in K$ for every $i, j$ and $c, e \in K$. It follows that $(-c+1) x_{1}{ }^{k_{i}+2}+\cdots+c=0$, which is a contradiction with the fact that $c \neq 0$. Therefore, none of the these equations has a solution in $K\left[x_{1}\right]$. By theorem 3.6, we have that $K\left[x_{1}, \ldots, x_{n}\right]$ is $d$-simple.

4. A differential criterion for maximality. In this section we establish a criterion for the ideal $A_{n}(d+\gamma)$ to be maximal in terms of polynomial solutions of a finite system of partial differential equations over the polynomial ring $K\left[x_{1}, \cdots, x_{n}\right]$. Our result generalizes and strengthens a theorem of Bratti and Takagi ([2]).

THEOREM 4.1. Let $d=\partial_{1}+\alpha_{2} \partial_{2}+\cdots+\alpha_{n} \partial_{n}$ be a Shamsuddin derivation of $K\left[x_{1}, \ldots, x_{n}\right]$, where $\alpha_{i}\left(x_{1}, x_{i}\right)=a_{i}\left(x_{1}\right) x_{i}+b_{i}\left(x_{1}\right) \in K\left[x_{1}, x_{i}\right], i=2, \ldots, n$. Let $\gamma \in$ $K\left[x_{1}, \ldots, x_{n}\right]$.

(a) If $A_{n}(d+\gamma)$ is a maximal left ideal of $A_{n}$, then the following conditions are satisfied:

(i) $\partial_{1}(v)-a_{i} \cdot v \neq b_{i}$, for every $v \in K\left(x_{1}\right), 2 \leq i \leq n$.

(i') $\partial_{1}(v)-a_{i} \cdot v \neq b_{i}$, for every $v \in K\left[x_{1}\right], 2 \leq i \leq n$.

(ii) $d(u)+a_{i} \cdot u \neq \partial_{i}(\gamma)$, for every $u \in K\left(x_{1}, \ldots, x_{n}\right), 2 \leq i \leq n$.

(ii') $d(u)+a_{i} \cdot u \neq \partial_{i}(\gamma)$, for every $u \in K\left[x_{1}, \ldots, x_{n}\right], 2 \leq i \leq n$.

(b) Reciprocally, suppose that conditions ( $i$ ) and (ii) are satisfied and moreover that $a_{i} \neq a_{j}$, for every $i \neq j$. Then, $A_{n}(d+\gamma)$ is a maximal left ideal of $A_{n}$.

Proof. (a): (i'): Since $A_{n}(d+\gamma)$ is a maximal left ideal, it follows from corollary 3.3 that $K\left[x_{1}, \ldots, x_{n}\right]$ is $d$-simple. Then, by theorem $3.6, K\left[x_{1}, x_{i}\right]$ is $\left.d\right|_{K\left[x_{1}, x_{i}\right]}$-simple, for every $i=2, \ldots, n$. By Shamsuddin's theorem ([3, Proposition 3.2]) we have that $\partial_{1}(v)-a_{i} \cdot v \neq b_{i}$, for every $v \in K\left[x_{1}\right], 2 \leq i \leq n$

(i): It follows from (i') and Lemma 3.5.

(ii'): Suppose that $p \in K\left[x_{1}, \ldots, x_{n}\right]$ satisfies $d(p)+a_{i} \cdot p=\partial_{i}(\gamma)$, for some $i \in$ $\{2, \ldots, n\}$. Let $R=\partial_{i}+p$. Then,

$$
[d+\gamma, R]=-a_{i} \partial_{i}+\left(d(p)-\partial_{i}(\gamma)\right)
$$

Hence,

$$
[d+\gamma, R]+a_{i} R=d(p)+a_{i} \cdot p-\partial_{i}(\gamma)=0 .
$$

Therefore, $R$ is a Darboux operator of $d+\gamma$ in $A_{n-1}\left[x_{1}\right]$. This is contrary to theorem 2.8 . 
(ii): We have noted already (proof of item (i')) that $d$ is simple derivation of $K\left[x_{1}, \ldots, x_{n}\right]$. Then, (ii) follows from (ii') and lemma 3.5.

(b): Let $R=\sum_{i_{2}+\cdots+i_{n}=0}^{N} P_{i_{2}, \ldots, i_{n}} \partial_{2}{ }^{i_{2}} \cdots \partial_{n}{ }^{i_{n}} \in A_{n-1}\left[x_{1}\right]$, where $P_{i_{2}, \ldots, i_{n}} \in K\left[x_{1}, \ldots\right.$, $x_{n}$ ], be an operator of $\operatorname{order} N$. Then, a simple calculation gives the following expression for $[d+\gamma, R]$ :

$$
\begin{aligned}
{[d+\gamma, R] } & =\sum_{i_{2}+\cdots+i_{n}=0}^{N}\left\{\left[d\left(P_{i_{2}, \ldots, i_{n}}\right)-i_{2} P_{i_{2}, \ldots, i_{n}} a_{2}-\cdots-i_{n} P_{i_{2}, \ldots, i_{n}} a_{n}\right] \partial_{2} i_{2} \cdots \partial_{n}{ }^{i_{n}}\right. \\
& +\left[-i_{2} P_{i_{2}, \ldots, i_{n}} \partial_{2}(\gamma)\right] \partial_{2}{ }^{i_{2}-1} \cdots \partial_{n} i_{n} \\
& +\cdots \\
& +\left[-i_{n} P_{i_{2}, \ldots, i_{n}} \partial_{n}(\gamma)\right] \partial_{2}{ }^{i_{2}} \cdots \partial_{n}{ }^{i_{n}-1} \\
& \left.+ \text { terms with order lower than }\left(i_{2}+\cdots+i_{n}\right)-1\right\} .
\end{aligned}
$$

Suppose that $N>0$. So, there exists $P_{j_{2}, \ldots, j_{n}} \neq 0$, for some $j_{2}+\cdots+j_{n}=N$. Without loss of generality we may suppose that $j_{2}>0$. Note that $\lambda_{j_{2}, \ldots, j_{n}}:=$ $d\left(P_{j_{2}, \ldots, j_{n}}\right)-j_{2} P_{j_{2}, \ldots, j_{n}} a_{2}-\cdots-j_{n} P_{j_{2}, \ldots, j_{n}} a_{n}$ is the coefficient of the monomial $\partial_{2}{ }^{j_{2}} \cdots \partial_{n}{ }^{j_{n}}$ in $[d+\gamma, R]$.

We consider

$$
R_{1}:=P_{j_{2}, \ldots, j_{n}}[d+\gamma, R]-\lambda_{j_{2}, \ldots, j_{n}} R .
$$

Evidently, $R_{1}$ has no term in $\partial_{2}{ }^{j_{2}} \cdots \partial_{n}{ }^{j_{n}}$, while the coefficient of the term $\partial_{2}{ }^{j_{2}-1} \cdots \partial_{n}{ }^{j_{n}}$ is the following:

$$
\begin{aligned}
q_{j_{2}-1, \ldots, j_{n}} & =P_{j_{2}, \ldots, j_{n}}^{2}\left\{d\left(\frac{P_{j_{2}-1, j_{3}, \ldots, j_{n}}}{P_{j_{2}, \ldots, j_{n}}}\right)+a_{2} \frac{P_{j_{2}-1, \ldots, j_{n}}}{P_{j_{2}, \ldots, j_{n}}}-j_{2} \partial_{2}(\gamma)\right. \\
& \left.-\left(j_{3}+1\right) \frac{P_{j_{2}-1, j_{3}+1, \ldots, j_{n}}}{P_{j_{2}, \ldots, j_{n}}} \partial_{3}(\gamma)-\cdots-\left(j_{n}+1\right) \frac{P_{j_{2}-1, \ldots, j_{n}+1}}{P_{j_{2}, \ldots, j_{n}}} \partial_{n}(\gamma)\right\} .
\end{aligned}
$$

We will analyze two cases.

FIRST CASE: If $P_{j_{2}-1, j_{3}+1, \ldots, j_{n}}=P_{j_{2}-1, j_{3}, j_{4}+1, \ldots, j_{n}}=\cdots=P_{j_{2}-1, j_{3}, \ldots, j_{n}+1}=0$.

We claim that $R_{1} \neq 0$. Indeed, in this case, (12) simplifies and the coefficient of the term $\partial_{2}{ }^{j_{2}-1} \cdots \partial_{n}^{j_{n}}$ is

$$
q_{j_{2}-1, \ldots, j_{n}}=P_{j_{2}, \ldots, j_{n}}^{2} j_{2}\left(d\left(\frac{P_{j_{2}-1, j_{3}, \ldots, j_{n}}}{j_{2} P_{j_{2}, \ldots, j_{n}}}\right)+a_{2} \frac{P_{j_{2}-1, \ldots, j_{n}}}{j_{2} P_{j_{2}, \ldots, j_{n}}}-\partial_{2}(\gamma)\right),
$$

which is non-zero by hypothesis.

Therefore, the ideal $A_{n}(d+\gamma)+A_{n} R$ contains a nonzero element $R_{1}$ without the term $\partial_{2}{ }^{j_{2}} \cdots \partial_{n}{ }^{j_{n}}$, and clearly $R_{1}$ does not have any monomial of order $N$ that was not already a monomial of $R$.

SECOND CASE: If $P_{j_{2}-1, \ldots, j_{k}+1, \ldots, j_{n}} \neq 0$, for some $k, 3 \leq k \leq n$.

Note that

$$
\begin{aligned}
\mu_{j_{2}-1, \ldots, j_{k}+1, \ldots, j_{n}}:= & d\left(P_{j_{2}-1, \ldots, j_{k}+1, \ldots, j_{n}}\right)-\left(j_{2}-1\right) P_{j_{2}-1, \ldots, j_{k}+1, \ldots, j_{n}} a_{2}-\ldots \\
& -\left(j_{k}+1\right) P_{j_{2}-1, \ldots, j_{k}+1, \ldots, j_{n}} a_{k}-\cdots-j_{n} P_{j_{2}-1, \ldots, j_{k}+1, \ldots, j_{n}} a_{n}
\end{aligned}
$$

is the coefficient of the term $\partial_{2}^{j_{2}-1} \cdots \partial_{k}^{j_{k}+1} \cdots \partial_{n}^{j_{n}}$ in $[d+\gamma, R]$. 
Consider

$$
R_{2}:=P_{j_{2}-1, \ldots, j_{k}+1, \ldots, j_{n}}[d+\gamma, R]-\mu_{j_{2}-1, \ldots, j_{k}+1, \ldots, j_{n}} R
$$

Evidently, $R_{2}$ has no term in $\partial_{2}^{j_{2}-1} \cdots \partial_{k}^{j_{k}+1} \cdots \partial_{n}^{j_{n}}$, while the coefficient of the term $\partial_{2}^{j_{2}} \cdots \partial_{k}^{j_{k}} \cdots \partial_{n}^{j_{n}}$ is the following:

$$
\xi_{j_{2}, \ldots, j_{n}}=P_{j_{2}-1, \ldots, j_{k}+1, \ldots, j_{n}}^{2}\left(d\left(\frac{P_{j_{2}, \ldots, j_{n}}}{P_{j_{2}-1, \ldots, j_{k}+1, \ldots, j_{n}}}\right)+\left(a_{k}-a_{2}\right) \frac{P_{j_{2}, \ldots, j_{n}}}{P_{j_{2}-1, \ldots, j_{k}+1, \ldots, j_{n}}}\right) .
$$

Now, by hypothesis and theorem 3.6, $d$ is a simple derivation of $K\left[x_{1}, \ldots, x_{n}\right]$. Applying lemma 3.5 and noticing that $a_{2} \neq a_{k}$, we obtain that $\xi_{j_{2}, \ldots, j_{n}}$ is non-zero. Then, the coefficient of the term $\partial_{2}^{j_{2}} \cdots \partial_{n}^{j_{n}}$ in $R_{2}$ is non-zero, while its coefficient of the term $\partial_{2}^{j_{2}-1} \ldots \partial_{k}^{j_{k}+1} \ldots \partial_{n}^{j_{n}}$ is zero. Repeating this argument, for every $k=3, \ldots, n$ such that $P_{j_{2}-1, \ldots, j_{k}+1, \ldots, j_{n}} \neq 0$, we obtain a non-zero element $\tilde{R} \in A_{n}(d+\gamma)+A_{n} R$ such that its coefficient of $\partial_{2}^{j_{2}} \cdots \partial_{n}^{j_{n}}$ is non-zero while all the coefficients of $\partial_{2}^{j_{2}-1} \cdots \partial_{k}^{j_{k}+1} \cdots \partial_{n}^{j_{n}}$, for $3 \leq k \leq n$, are zero. We are back to the first case.

In any case, the ideal $A_{n}(d+\gamma)+A_{n} R$ contains a nonzero element $\tilde{Q}$ with no monomial $\partial_{2}{ }^{j_{2}} \cdots \partial_{n}{ }^{j_{n}}$ and whose monomials of order $N$ are among the monomials of $R$. Note that, by (11), any element of the form $f\left(x_{1}, \ldots, x_{n}\right)[d+\gamma, \tilde{Q}]+g\left(x_{1}, \ldots, x_{n}\right) \tilde{Q}$, where $f\left(x_{1}, \ldots, x_{n}\right), g\left(x_{1}, \ldots, x_{n}\right) \in K\left[x_{1}, \ldots, x_{n}\right]$, does not have the term $\partial_{2}{ }^{j_{2}} \cdots \partial_{n}{ }^{j_{n}}$ either.

Proceeding in this way, we can eliminate all the monomials of positive order and we get a non-zero element with order zero, that is

$$
\left(A_{n}(d+\gamma)+A_{n} R\right) \cap\left(K\left[x_{1}, \ldots, x_{n}\right] \backslash\{0\}\right) \neq \emptyset .
$$

Now, let

$$
P=\sum_{i_{2}+\cdots+i_{n}=0}^{N} P_{i_{2}, \ldots, i_{n}} x_{2}{ }^{i_{2}} \cdots x_{n}^{i_{n}} \in\left(A_{n}(d+\gamma)+A_{n} R\right) \cap\left(K\left[x_{1}, \ldots, x_{n}\right] \backslash\{0\}\right),
$$

where $P_{i_{2}, \ldots, i_{n}} \in K\left[x_{1}\right]$. Notice that, since $P$ is a polynomial, $[d+\gamma, P]=d(P)$. Then, repeating the argument of the proof of theorem 3.6, (ii) $\Rightarrow$ (i), we see that

$$
\left(A_{n}(d+\gamma)+A_{n} R\right) \cap\left(K\left[x_{1}\right] \backslash\{0\}\right) \neq \varnothing .
$$

Therefore, $A_{n}(d+\gamma)+A_{n} R=A_{n}$. By lemma 2.4, $A_{n}(d+\gamma)$ is a maximal left ideal of $A_{n}(K)$.

EXAMPLE 4.2. Let $d$ be a Shamsuddin derivation of $K\left[x_{1}, \ldots, x_{n}\right]$ and $\gamma \in$ $K\left[x_{1}, \ldots, x_{n}\right]$. If $\operatorname{deg}_{x_{i}}(\gamma)=0$, for some $i \in\{2, \cdots, n\}$, then $A_{n}(d+\gamma)$ is not a maximal left ideal of $A_{n}$. Indeed, in this case, the equation $d(u)+a_{i} \cdot u=\partial_{i}(\gamma)$ has $u=0$ as a solution.

We show next that the conditions $a_{i} \neq a_{j}$, for $i \neq j$, in part (b) of theorem 4.1 cannot be dropped in general.

EXAMPLE 4.3. Let $d=\partial_{1}+\left(a x_{2}+b_{2}\right) \partial_{2}+\left(a x_{3}+b_{3}\right) \partial_{3}$ be a simple Shamsuddin derivation of $K\left[x_{1}, x_{2}, x_{3}\right]$ with $\operatorname{deg} a \geq 1$. Let $\gamma:=x_{2}+x_{3}$. Then,

(1) Conditions (i) and (ii) of Theorem 4.1(a) are satisfied.

(2) $A_{3}(d+\gamma)$ is not a maximal left ideal of $A_{3}$. 
Proof. (1): By theorem 3.6, to say that $d$ is simple is equivalent to say that the equations $\partial_{1}(v)-a_{i} \cdot v=b_{i}, i=2, \cdots, n$, have no solution in $K\left(x_{1}\right)$. Then, condition (a)(i) of theorem 4.1 is satisfied. Now we consider condition (a)(ii). By lemma 3.5 and the fact that $d$ is simple, this is equivalent to condition (a)(ii').

Suppose that there exists $u \in K\left[x_{1}, x_{2}, x_{3}\right]$ such that

$$
d(u)+a \cdot u=1 .
$$

Let $i \in\{2,3\}$. Applying $\partial_{i}$ to (13) we have,

$$
\partial_{i}(d(u))=-a \partial_{i}(u) .
$$

Hence,

$$
\begin{gathered}
d\left(\partial_{i}(u)\right)+a \partial_{i}(u)=-a \partial_{i}(u), \\
d\left(\partial_{i}(u)\right)=-2 a \partial_{i}(u) .
\end{gathered}
$$

Therefore $\partial_{i}(u) \in K$ since $d$ is a simple derivation of $K\left[x_{1}, x_{2}, x_{3}\right]$. Then $d\left(\partial_{i}(u)\right)=0$ and $\partial_{i}(u)=0$, since $a \neq 0$.

Since this is valid for $i=2,3$, we obtain that $u \in K\left[x_{1}\right]$. Then, (13) becomes

$$
u^{\prime}=-a u+1 \text {. }
$$

This is absurd since $\operatorname{deg} a \geq 1$.

(2): Let $R:=\partial_{2}-\partial_{3}$. We have $[d+\gamma, R]=-a R \in K\left[x_{1}, x_{2}, x_{3}\right] R$. Thus, by theorem 2.8, $A_{3}(d+\gamma)$ is not a maximal left ideal of $A_{3}$.

REMARK 4.4. Simple Shamsuddin derivations of $K\left[x_{1}, x_{2}, x_{3}\right]$ with $a_{2}=a_{3}$ exist. For example, $d=\partial_{1}+\left(x_{1}^{2} x_{2}+x_{1}^{3}\right) \partial_{2}+\left(x_{1}^{2} x_{3}+x_{1}+1\right) \partial_{3}$ is one of them. (See [7]).

\section{REFERENCES}

1. J. Bernstein and V. Lunts, On non-holonomic irreducible D-modules, Invent. Math. 94 (1988), 233-243.

2. G. Bratti \& M. Takagi, Differential equations and maximal ideals on the Weyl algebra $A_{2}(\mathbb{C})$, Rend. Sem. Mat. Univ. Padova, 107 (2002), 209-223.

3. S. C. Coutinho, $d$-Simple rings and simple D-modules, Math. Proc. Camb. Phil. Soc. 125 (1999), 405-415.

4. S. C. Coutinho, A primer of algebraic D-modules, London Mathematical Society Student Texts No. 33 (Cambridge University Press, 1995).

5. A. M. de S. Doering, Y. Lequain and C. C. Ripoll, Differential simplicity and cyclic maximal ideals of the Weyl algebra $A_{2}(K)$, preprint.

6. K. R. Goodearl and R. B. Warfield Jr., Krull dimension of differential operators rings, Proc. London Math. Soc. (3) 45 (1982), 49-70.

7. Y. Lequain, Shamsuddin derivations of $K\left[x_{1}, \ldots, x_{n}\right]$, preprint. $531-554$.

8. V. Lunts, Algebraic varieties preserved by generic flows, Duke Math. J. 58 (1989),

9. A. Maciejewski, J. Moulin-Ollagnier and A. Nowicki, Simple quadratic derivations in two variables, Comm. Algebra 29 (2001), 5095-5113.

10. A. Shamsuddin, Ph.D. Thesis, University of Leeds (1977).

11. J. T. Stafford, Non-holonomic modules over Weyl algebras and enveloping algebras, Invent. Math. 79 (1985), 619-638. 